\title{
Alzheimer's disease: is a cure possible?
}

\author{
Byron AK* \\ Faculty of Health and Medical Sciences, Perron Institute for Neurological and Translational Science, Australia
}

\begin{abstract}
Alzheimer's disease $(\mathrm{AD})$ is the commonest cause of dementia in the world. The dementia of $\mathrm{AD}$ is referred to as "global "as it affects all higher neurological functions which deteriorate progressively over time. $\mathrm{AD}$ may begin at any age, even in childhood, with the incidence increasing as years pass so that $\mathrm{AD}$ becomes very prevalent after the age of 60 years when it is estimated that $6 \%$ of the population are affected. As rule of thumb, the incidence of $\mathrm{AD}$ increases by about $1 \%$ for every year from then on i.e. it is estimated that by the age of 80 years $26 \%$ of the population will suffer from the disorder. Thus, $\mathrm{AD}$ poses an enormous problem worldwide, medically, socially and economically and it will become more so as life expectancy increases in the future.

Much is written in the lay and scientific literature about the possibility of a cure for AD particularly about the potential for stem cells to replace lost neurons from the cerebral cortex. As will be seen below this may be an unrealistic expectation because by the time dementia is clinically manifest the cerebral cortex is in a state of advanced atrophy with enormous loss of neurons. Unfortunately, a great deal of the research on AD seems to overlook or be unaware of this fact.
\end{abstract}

\section{The higher neurological functions}

The cerebral cortex, the grey matter of the brain, is responsible for all higher neurological functions such as consciousness, memory, emotion, cognition, intelligence, ideation and abstract thought, volition, motor control, bodily sensation, speech, musical appreciation, vision and hearing. Furthermore, the traits which define an individual's personality reside in the cerebral cortex. Although localised cortical centres exist for such faculties as speech, hearing and vision the brain works mainly as whole. In $\mathrm{AD}$ all of these higher intellectual activities are severely compromised and in the final stages of dementia only the vegetative functions remain with the by now bedridden patient dying of hypostatic pneumonia.

\section{Neuropathology}

First and foremost is the degeneration and outfall of cortical neurons, a process which begins in the hippocampus of the temporal lobes extending to the frontal lobes and from there throughout the brain? Degenerating neurons contain neurofibrillary tangles composed of tau protein. In $\mathrm{AD}$ the affected neurons shrink and disintegrate with the cellular debris taken up by pleomorphic microglia. Astrocytic gliosis follows the neuronal loss. The neuronal changes are the essential features of the neuropathology of $\mathrm{AD}$, the outfall of neurons correlating with the intellectual decline and progressive dementia. At the end stage the brain in $\mathrm{AD}$ appears severely shrunken to the naked eye, the sulci are widened, and the gyri narrowed. A human brain normally weighs in the vicinity of 1500 grams. In $\mathrm{AD}$ brain weight is greatly reduced often to less than 1000 grams. AD is caused by a yet unknown mechanism but with a hint that the aetiology and pathogenesis may involve altered glucose metabolism [1].

Associated with the neuronal changes is the accumulation of amyloid material in the interstitium of the cerebral cortex. The amyloid is in the form of discrete "senile" plaques composed of A $\beta$ amyloid protein which is in the shape of densely folded $\beta$ pleated sheets. Some theories maintain that the A $\beta$ amyloid is toxic to neurons, but this effect has not been convincingly substantiated scientifically in vivo. The amyloid may well be a by-product of the degenerative process as it is in other pathological contexts.

\section{The requirements for a cure of alzheimer's disease}

From what is known of the neuropathology it can be reasonably appreciated that a cure for $\mathrm{AD}$ would require the replacement of neurons and their connections in the cerebral cortex and beyond. Simple replacement of lost neurons by differentiated stems cells would not be sufficient alone to restore lost intellectual functions because the appropriate network of neuronal connections would need to be also reproduced.

There are two methods by which neuronal connections develop in the growing brain. Firstly, there are the 'preordained' networks within the recognised centres such as Broca's area for speech in the left cerebral hemisphere or the visual cortex in the occipital lobes. During development the neurons in the special centres arrange themselves from 'inherent messages' so that their 'preordained' relationships are ready to receive the relevant external stimuli and thereby acquire the appropriate function such as speech or sight.

The second mechanism of neuronal organisation is much more flexible and is dependent upon experience and use. The millions of neurons in the remaining vast areas of the cerebral hemispheres outside of the recognised centres arrange their connections from signals received from the internal and external environment related to experience and learning, a process which begins in the foetus and continues in infancy and childhood and to a lesser extent throughout life. Thus, some functions are inherent with fixed connections such as in the centre for gait in the lumbar spinal cord, while others like

${ }^{\star}$ Correspondence to: Byron AK, Faculty of Health and Medical Sciences, Perron Institute for Neurological and Translational Science, Australia, Email: byron. kakulas@perron.uwa.edu.au

Received: October 16, 2018; Accepted: October 26, 2018; Published: October 29,2018 
language are learned in which case the organisation of synapses is a response to external stimulation.

A remarkable property of the human brain is that it can function and grow simultaneously as occurs in early life. Although brain development mainly takes place during childhood the fine architecture of the brain continues to alter and modify throughout life according to use. It is these continuously changing neuronal connections and their networks which underlie the modalities of consciousness, selfawareness, personality, memory, emotion, cognition, language, other learned activities and manual skills. Some of these functions are represented in particular lobes of the brain e.g. planning and foresight are located in the frontal lobes, memory and emotion are within the temporal lobes, body image and spatial orientation are found in the parietal lobes and vision is located in the occipital lobes. Nevertheless, the regions responsible for specific functions can vary according to use or disease so that they may be found in quite different parts of the brain following destructive lesions e.g. after a stroke or trauma. The process of continuous change is active on a daily basis and is referred to as cerebral plasticity.

Because Alzheimer's disease is a widespread condition, affecting all of the cerebral cortex of both hemispheres, in order to provide a cure, it would be necessary to put in place a very large number of neurons and reproduce the networks responsible for specific functions. Therefore, to bring about a cure not only are millions of new cortical neurons required but also their proper connections must be reproduced. Immunologically compatible stem cells transplanted into the cerebral cortex may differentiate into neurons, but they would also need to recreate the complex network of neurites and synapses organised in an exactly similar fashion as the networks which have naturally evolved over a lifetime in order to restore lost functions and cure the dementia.

\section{Prevention}

A more realistic approach in avoiding the impending 'epidemic' of Alzheimer's Disease is by means of prevention. It is a remarkable finding, in recent years, that the lifestyle changes recommended for the prevention of coronary heart disease also apply to the brain. Regular physical exercise is the most important of these followed by a healthy natural diet rich in antioxidants. The Mediterranean cuisine which includes lots of nuts, fresh fruit, vegetables and olive oil is ideal in this respect [2].

\section{Conclusion}

It possible that in the foreseeable future the basic cause of $\mathrm{AD}$ will be discovered. Clues to the processes which may be involved are the genetic risk factors such as the APOE4 genotype and the TOMM 40 gene which are known to predispose to the disease $[3,4]$. When the molecular aetiopathogenesis of $\mathrm{AD}$ is discovered, it may be possible to arrest its progression. In the meanwhile, research projects should be undertaken in accordance with the neuropathology of $\mathrm{AD}$. This advice is important not only to avoid the waste of time, money and energy but also to focus resources on what is a feasible research plan. Until then a healthy lifestyle is recommended especially for the young so that the incidence of $\mathrm{AD}$ will be substantially diminished in the later years of life.

\section{References}

1. Tai J, Liu W, Li Y, Li L, Holscher C (2018) Neuroprotective effects of a triple GLP-1/ GIP/glucagon receptor agonist in the APP/PSI transgenic mouse model of Alzheimer's disease. Brain Research 1678: 64-74. [Crossref]

2. Martins R, Pan M (2013) Understanding Alzheimer's: the complete Australian guide to the management and prevention Alzheimer's.

3. Roses $\mathrm{AD}$ (2006) On the discovery of the genetic association of apolipoprotein E genotypes and common late-onset. Alzheimer disease. J Alzheimer Dis 9: 361-366. [Crossref]

4. Roses AD, Lutz MW, Amrine-Madsen H, Saunders AM, Crenshaw DG, et al. (2010) A TOMM40 variable-length polymorphism predicts the age of late-onset Alzheimer's disease. J Pharmacogenomics 10: 375-384. [Crossref]

Copyright: (C2018 Byron AK. This is an open-access article distributed under the terms of the Creative Commons Attribution License, which permits unrestricted use, distribution, and reproduction in any medium, provided the original author and source are credited. 